\section{CASE REPORT: ACASE OF DOUBLE INFERIOR VENA CAVA}

\author{
Rampada Sarker'1, Asif Rahim², Anisuzzaman², S M Mahbubur \\ Rahman², Sarwar Kamal $^{2}$, S K A Razzaque ${ }^{3}$, Suman Nazmul \\ Hosain 4
}

\section{Introduction}

Major anomaly involving Inferior Vena Cava is rare in clinical practice. But variation in normal anatomy of IVC is reported in up to $3 \%$ of the population ${ }^{1}$. A double inferior vena cava occurs when the left sacrocardinal vein fails to loose its connection with the left subcardinal vein. Left superior vena cava which is persistent anterior cardinal vein also drains into Right atrium by the way of coronary sinus. More rarely right and left inferior vena cava ascend separately and enter the right atrium individually. In this variant of anomaly both IVC ascend posterior to liver. The IVC on the Rt receives the Rt portal vein, one of the left portal veins and the Rt hepatic vein. The Left IVC receives one of the left portal veins and the left hepatic vein. In case of partial anomalous pulmonary venous drainage (PAPVD), one or more of the pulmonary veins drain into the Rt atrium. So, in addition to SVC, IVC and coronary sinus any extra vessel draining into Rt atrium may make diagnostic confusion. In this case we diagnosed and managed a case of double IVC per-operatively.

\section{Case Report}

A young lady named Miss R. A. aged 18 years and a student from Dinajpur, Bangladesh was admitted in the department of cardiac surgery, NICVD, Dhaka under SU-7 for surgical closure of atrial septal defect. All preoperative investigation for open heart surgery was within normal limits. The disease was confirmed previously by color doppler echocardiogram and cardiac catheterization. The patient underwent surgery in 2008. Following standard NICVD protocol after induction of general anesthesia, median sternotomy was done. After pericardiotomy and sling placement, systemic venous drainage or any other congenital anomaly were searched as a routine protocol. There were two vessels of nearly equal diameter at the usual site of Inferior Vena Cava apparently draining into Right atrium. Two pulmonary veins (Rt) were seen draining into Lt atrium. To remove diagnostic dilemma, blood sample were sent from both the vessels for gas analysis. The sample results were same and

1. Asst Professor, Cardiac Surgery, NICVD, Dhaka

2. Asst Registrar, Cardiac Surgery, NICVD, Dhaka

3. Associate Professor, Pediatric Cardiology, NICVD, Dhaka

4. Associate Professor, NICVD, Dhaka very similar to usual systemic venous sample. Both the vessels were cannulated and snugged along with SVC to establish cardiopulmonary bypass. After cross clamping and cardioplegic arrest of heart, right atrium was opened and it was found that both of those vessels have opening into the cavity of right atrium. The channel in relatively left position was a little bigger than the right one. When the venous cannula draining the left channel was momentarily clamped and tape around the right channel loosened, there was profuse leakage around the cannula draining the right confirming communication between them. During this maneuver, there was no change in coronary sinus drainage or from the opening of the pulmonary veins in the left atrium.

The atrial septal defect was closed with glutaraldehyde treated pericardial patch leaving SVC, Coronary sinus and both the IVCs draining in the right atrial cavity and the pulmonary veins draining on the left. Cross clamp was released and right atrium closed. Blood sample from RA sent for oxymetry revealed no residual shunt The heart was weaned from CPB. After proper heamostasis, chest was closed. The postoperative recovery was uneventful.

A postoperative color Doppler echocardiogram was done on $6^{\text {th }}$ POD. There was no residual shunt. The presence of double IVC was also checked. It was found that within the hepatic tissue two IVCs proceeded to drain into right atrium. The patient had a smooth postoperative recovery and on $8^{\text {th }}$ POD she was discharged from the hospital. She came for follow up after one and three months with smooth recovery trends.

\section{Discussion}

Errors in the embryological development of the inferior vena cava (IVC), although rare, can result in several anomalies. Congenital variations of the IVC originate during $4^{\text {th }}$ to $8^{\text {th }}$ week of embryogenesis of three paired veins, namely (1) posterior cardinal veins, (2) subcardinal veins, and (3) supracardinal veins ${ }^{1}$. Variations of the IVC may occur in $3 \%$ of the population. A complex process of embryogenesis forms the IVC during 6th - 10th week of gestation. Improper completion of the process may result in 4 anatomic anomalies :- duplication of IVC, transposition of left sided IVC, retroaortic left renal vein and circumaortic left renal vein ${ }^{2}$. Preoperative diagnosis of the anomalies shown reduce the complication rate of abdominal aortic operations ${ }^{3}$.

In practice double IVC is an anomaly rarely encountered during clinical practice. There is report of a case with double IVC who developed thrombosis of the left IVC following an electrophysiologic procedure, which resulted in an 
asymptomatic pulmonary embolism ${ }^{4}$. Variations of the IVC are diagnosed in routine dissection studies, in retroperitoneal surgeries, in radiological studies and with its magnetic resonance imaging findings ${ }^{5}$. Among the other imaging techniques, computerized tomography is a non-invasive, effective technique for diagnosing diseases of the retroperitoneal space and, particularly, for detecting anomalies of the main vessels, such as the aorta or IVC, in asymptomatic patients ${ }^{6}$. Double IVC is also often reported by the transplant urologist during renal transplantation procedures ${ }^{7}$. Hematuria may be the presenting feature due to congestion when an abnormal vein such as double IVC or left IVC crosses over the aorta ${ }^{8}$.

In our case of ASD repair, Rt. atrium had to be opened with proper cannulation, snaring and snugging systemic great veins, i.e. in this case the SCV and both the IVCs. Presence of an extra vein draining into Rt atrium was creating dilemma. To exclude Schimiter syndrome and to confirm the additional vein is systemic in nature, we loosened the snare around the extra vein (Rt IVC) and momentarily clamped the main (left, here) IVC. Immediately after clamping there was a gush of blood coming around the additional vein confirming a free communication of between them. The ABG analysis of the sample sent from both the veins were same and similar to usual IVC sample. All these confirmed the systemic venous nature of both the IVCs. We closed the ASD leaving the SVC, both the IVCs and tricuspid valve on the right atrial side and the pulmonary vein openings and te mitral valve on the left atrial side.

There was no rise of hepatic enzymes postoperatively, so no hepatic congestion. Postoperative color Doppler revealed double inferior vena cava. The other question remains why it was missed during color Doppler and cardiac catheterization. Perhaps the operators missed because of its rarity, the presence of double inferior vena cava was out of mind. Postoperatively presence of double inferior vena cava was easily diagnosed because the cardiologist emphasized on its presence.

\section{Conclusion}

Presence of double inferior vena cava into Rt atrium can be easily managed during open heart surgery if it can be diagnosed even peroperatively. Both IVC's should be cannulated and snared during cardiopulmonary bypass to prevent airlock and poor venous drainage. Moreover inattention during pericardial patch closure of ASD may accidentally cover coronary sinus opening under patch by taking accessory IVC opening into Rt atrium as coronary sinus opening. We reported this case because its incidence is rare and color Doppler echocardiogram and cardiac catheterization may miss its presence in other patients.

\section{References:}

1. Milloy FJ, Anson BJ, Cauldwell EW. Variations in the IVC and their renal and lumbar communications. Surg Gynecol Obstet. 1962, 115:131.

2. Palit, S., Deb, S. A Rare Presentation of Double Inferior Vena Cava with Anomalous Pattern Of Azygos And Hemiazygos Venous Systems : A Case Report. J Anat. Soc. India 51(1) 65-67 (2002)

3. Giordano, J.M; Trout, H.H; (1986): Anomalies of inferior vena cava; Journal of Vascular Surgery. 3 (6) : 924-8

4. Zubair A. Hashmi, MD, Gregory G. Smaroff, MD. Dual Inferior Vena Cava: Two Inferior Vena Cava Filters. Ann Thorac Surg 2007; 84:661-663.

5. Rohrer M, Cutler B. Placement of two Greenfield filters in a duplicated vena cava. Pub Med. Surgery 1988;104:572574.

6. Saito H, Sano N, Kaneda I, et al. Multisegmental anomaly of the inferior vena cava with thrombosis of the left inferior vena cava Cardiovasc Intervent Rad 1995;18:410-413.

7. Tatsuya Nakatani, Taku Kim, Toshihide Naganuma, Junji Uchida, Yoshiaki Takemoto, Kazunobu Sugimura. Kidney Transplants from LivingRelated Donors Having Double Inferior Vena cava. Urol Int 2004;72:358-360.

8. Takashi K, Shunsaku F, Shimoaraiso Y, Idesako T.Double IVC found by macroscopic hematuria. Nishinion journal of urology. 2006, Vol 68 No 3 Page 126-129. 